\title{
INFERTILITY: CULTURAL DIMENSIONS AND IMPACT ON WOMEN IN SELECTED COMMUNITIES IN KENYA
}

\author{
VIOLET KIMANI \& JOYCE OLENJA \\ Department of Community Health \\ University of Nairobi \\ Box 19676 Nairobi, Kenya \\ Email: Olenja@comblth.ac.ke
}

\section{ABSTRACT}

Infertility is a growing problem in Africa and affects the lives of many couples. As a health problem, it is largely culturally and socially constructed in such a way that even though it affects a couple it is the woman who bears the burden. This perspective has major implications for women whose status hinges on fertility performance. This paper presents the cultural construction of infertility and how it impacts on the lives of infertile women in Kenya. The presentation is based on material collected through qualitative methodologies among infertile and fertile women as well as through key informant interviews. Overall, infertility not only erodes the status of infertile women but also threatens their source of livelihood as some of their verbatim comments vividly point out. The burden of infertility is compounded by the fact that at the national programme level it is underplayed as a problem, the main focus being on fertility control. This is a situation that needs to be redressed so that those who are infertile, regardless of their numbers, receive the attention they require to experience quality life.

\section{INTRODUCTION}

ver the past years, bio-medical and social sciences have contributed significantly to the exploration of human reproduction. This interest in reproduction is evident in a number of edited volumes on fertility, gender and decision making, family planning, and population politics (MacCormack 1994, Ginsburg and Rapp 1995, Greenhalgh 1995) and more recently, cross-cultural analysis of childbirth '(Handwerker 1990, Davis-Floyd and Sargent; 1997). Comparatively, infertility, as a subject of study, has not received as much attention as fertility. Many population programs particularly in developing countries have invested résources in the study of and 
control of fertility. This emphasis is based on the premise that the rate of natural population increase, resulting from high fertility, is inimical to economic development. Conversely, economic development can only be achieved through control and subsequent reduction in fertility. In this context, infertility has, for a long time been relegated to the background to the extent that the numbers affected remains only speculative. However, awareness to the age-old problem of infertility is now growing (Whiteford and Gonzalez, 1995; Inhorn and Buss, 1993 Inhorn, 1994).

Infertility is a problem that affects men and women every where in the world. Although estimates of its prevalence are not precise and vary from region to region, about $8 \%$ of couples experience some form of infertility problem during their reproductive lives. When extrapolated to the global population, this means that $50-80$ million people probably have a problem with fertility, a condition that causes personal suffering and disruption to social and family life. It is estimated that there are about two million new infertile couples per year, and the numbers are increasing (Rowe P. J. quoted in WHO Manual, 1993)

Leke et al (1993) discuss the impact of cultural, environmental, and socio-economic factors on reproductive health and infertility in subSaharan Africa, and Latin America. The study notes that in Kenya infertility results from the high incidence of sexually transmitted diseases. Karanja (1982), reported that infertility is a major problem in East Africa, and estimated that in the major urban centres of East Africa, up to one third of all women would be sterile by the age of 30 years. According to Mati, Anderson, Carty and McGlashan, (1987) about two thirds of the clinical time at the Kenyatta National Hospital which is the main referral hospital is spent on infertility cases. Most of these cases have blocked tubes due to chronic pelvic inflammatory disease (Karanja 1982); attesting to the role of infections in precipitating secondary infertility (Plummer et al. 1987)

In their study of patterns and predictors of infertility among African women in 27 African countries, Ericksen and Brunette, (1996), estimated national infertility prevalence in Kenya to be around $11.9 \%$, with Western and Coast provinces having the highest rates. This is by no means a small number of people, bearing in mind that approximately $36 \%$ of Kenya's 27 million people fall within the reproductive age bracket (KDHS, 1993). Since then the population has grown to 29 million (1999 National Population Census). It is therefore important to note that at the national level, Kenya has now adopted a reproductive health strategy to guide the implementation of a 
comprehensive reproductive health programme which includes, among others, Management of Infertility and Reproductive Health Research. (MOH, 1996). The inclusion of infertility management in the Kenyan National Reproductive Health Strategy document for the years 1997 to 2010 underscores the importance the government attaches to the problem of infertility, that has far reaching psychological, social, and economic consequences, especialiy for women.

\section{The Context of infertility}

In spite of the established findings that infertility is a couple problem, it is still largely viewed as a female problem; a construction that is largely rooted in culture so that the understanding of infertility becomes an exercise in explicating societal values erribedded in culture. In available studies, it is evident that fertility is of immense cultural significance in most communities, largely because it is linked to the very survival of the group. Fertility rites abound in these communities to ensure that procreation is guaranteed. For example, among the Aowin of Ghana, Ebin (1982) notes that fertility is defined in a woman's initiation rite and conversely, mediums view infertility as not only an individual malady but as the focal point of the client's relationship with the social universe. Erny (1969) reported that the desire for many children in traditional Africa is recognized as having a compelling social force. Women without children were pitied, feared, hated and ostracized. Three types of explanations of infertility were simultaneously entertained: some sort of anatomic or physiologic defect in the woman, some tribal ethic or taboo violated, or some metaphysical force or power interfering with conception and birth. Sterility was seldom attributed to the man and in most societies, it was and still, is a cause for divorce.

In Cameroon, Feldman-Savelsberg, 1994, in his paper: "Plundered kitchens and empty wombs: Fear of infertility in the Cameroonian grasslands", fertility and infertility are at the centre of ritual activities that involve both men and women and ultimately the king. However, when it fails, women assume the bulk of the responsibility as her womb "fails" to bring forth a child.

There are also studies that have focused on the impact of infertility on women. (Whiteford and Gonzalez, 1995; Inhorn and Buss, 1993; Inhorn, 1994). Initially, these may be surprise, denial, and isolation, followed by anger, guilt, depression, and even grief. Some of these, particularly stress and depression were reported in a study conducted 
in Greece (Tarlatzis, 1993). Also in Mozambique, the Macua infertile women experience exclusion from social activities and traditional ceremonies as a consequence of their infertility (Gerrits, 1997). A woman who, for one reason or other, is unable to give birth seems to undergo serious social stigma and this is deeply felt.

The cultural explanations of infertility have, in a way, been reinforced by the culture of medicine so that whereas there are relatively elaborate services for women there are very few dealing with male reproductive health issues. The approaches in scientific research have therefore inadvertently feminized infertility as well as legitimised the traditional belief that infertility is essentially a woman's problem. This, despite the fact that it is estimated that the man is responsible in $30 \%$ of the cases, the woman in approximately $40 \%, 20 \%$ in both and in about $10 \%$ no cause of infertility is identifiable even after thorough and complete evaluation. The WHO Multi-Centre study (1990) further confirms these clinical findings and places the male factor at $30 \%$ to $60 \%$. Thus, white infertility is a couple's problem, the cullural perception of infertility as a female problem is perpetuated by medical culture and practice which inadvertently leads to poor management strategies, biased outcomes, and sometimes misallocation of resources.

Materials used in this paper are based on research conducted among women by the authors over the years in various communities in Kenya. The data was collected principally through qualitative methodologies, specifically narratives and focus group discussions of infertile women and fertile women. An analysis of the data indicates that women view infertility largely as female, and almost unquestionirgly accept the blame. Similarly, men tend to relegate the problem of inferility to women and only in rare circumstances such as a curse do they attribute infertility to other realms that include men. While they may refer to pasi sexual behaviour of women as a likely cause of infertility this was rarely referred to as applicable to men.

\section{Marriage and the cultural significance of fertility}

Marriage, one form or the other, and childbearing occur so widely in the world that both are often regarded as universal. Motivations for childbearing are assumed not to be entirely unique to the individual but to consist of some of those elements plus a substantial component of culturally influenced desires (Arnold et al 1975). Because of the binding and unique nature of childbearing women feel that birthing wields 
immense power that is more noticeable in a society where women command far much less power than men in the public domain. This thesis has been argued elsewhere that reproduction is not only the one unique gender-related asset of women, but also the only power base that women in all social classes and marital relationships can more or less control. Women view reproduction not as an isolated episode but as an event which is integrated with other aspects of her life. Having a baby designates a new status and identity as well as define new roles. Because of the holistic way in which women view childbearing, the notion of successful reproduction is considerably more complex. Though, in almost all cases, the goal of the live birth of a healthy infant is paramount, success means, primarily, a satisfactory personal experience as motherhood becomes integrated with the rest of a woman's life (Kitzinger 1972).

Birthing is not just one of those procedures, it is a process that also confers power to women in any given society and even more so in more traditional societies. Perceived in this way, women, short of other sources of power, much of it is achieved via birthing- an arena where they feel that they are in complete control. Throughout the discussions with the women, it was evident that birthing is an area where women have power of thair own; a sphere that is impenetrable except by initiation. In the study sites, it was explicit that childbearing is highly valued by the women and that an infertile woman is considered very unfortunate and a social misfit among fellow women, as this is the one thing that binds womer together. To many women, this is their destiny. It is the pinnacle of motherhood. In Kakamega, among the Luhya, the research team was informed that during prayers, there is acknowledgement to. God for having enabled one to have children, thereby "removing the shame of childlessness". Similarly, among the traditional Agikuyu, there used to be prayers of thanksgiving to God for the blessings of wealth and fertility, the utmost blessings a couple can aspire to; the optimal level of human fulfillment. Here a couple with plenty of wealth but no children is considered as having nothing!

Thus, fertility is of immense cultural significance in most communities largely because it is linked to the very survival of the group. Fertility rites abound in these communities to ensure that procreation is guaranteed. Given the high regard accorded to fertility, infertility then constitutes a major problem both at the individual and societal levels. It disrupts the general rythymn of a woman's life. The cultural emphasis on women's status and childbearing makes infertility a major concern 
particularly for women. While there is growing recognition that infertility does occur in both men and women, the tendency is still to regard infertility as a female issue.

\section{Perceived Causes of Infertility}

Research findings in Kenya indicate that there is a wide range of causes attributed to infertility. These cover natural as well as socially generated causes. Failure to give birth in a society that attaches high value on children affects one negatively. Similarly there is hardly any sympathy for those who lose children during delivery. Among the Luhya, one is referred to in a derogatory manner as india avana; literally "the greedy baby eater. Among the Agikuyu, the constant loss of pregnancies and babies is often attributed to the woman's past promiscuity, including induced abortions. Such a woman receives very little sympathy, especially from her in-laws, who considered themselves the aggrieved party, as they feel cheated. In the Focus Group Discussions reference was made to personal behaviour, Sexually Transmitted Diseases and breach of taboos such as non-payment of bridewealth among the Kikuyu. Among the Luo, if a girl delivers at home while her mother is still fecund, the mother should not cook for her until she is over puerperium and gets her first period. If the mother cooks for her before this period is over, the girl will not conceive again, thus acquiring the state of secondary infertility. Among the Luhya if a girl delivers a child by a relative and keeps the baby, this will cause her infertility, and bring a curse to the other members of the family. To avoid an imminent calamity, the baby is killed immediately or given up for adoption. Here social etiquette and social control were central in defining modes of behaviour and social relations and boundaries.

More recent arguments attribute causes of infertility to the excessive use of contraceptives, (especially oral pills) before marriage or before conceiving the first child. Previous abortions, cancers or diseases of the reproductive tract were also cited. Among the Mijikenda of Kilifi, infertility is presently believed to be as a result of witchcraft and illicit brews (UNFPA/Population Council 2001).

Perceived factors in the inability to conceive ranged from the absence of menstrual blood altogether to menstrual blood of "wrong" quality and quantity. It was stated that blood that was too thick, too thin, too much, or too little was believed to be contra indicative to conceiving. A woman with 'cold' eggs, reflective of a weak body would be infertile. This is 
analogous to the belief in a Guatemala village that infertility was due to a cold womb which was not hot enough to receive the semen (Helman, 1994). Other factors included induced abortions, witchcraft and supernatural punishment. Varying time intervals constituted what was perceived as barrenness, ranging from one to ten years of childlessness in a woman living with a man constantly and trying to conceive. In some instances, it was explained that some women are simply formed "like males" and are therefore, physically unable to conceive. Further probing of the meaning of this revealed the implied absence of essential reproductive organs such as the uterus or ovaries, or, in some cases, advanced age. A woman who is "too fat" was said to "burn" her eggs with the excessive heat from body fat ${ }_{i}$ thus rendering them useless for conception. A woman who is "too thin" is said to be under-nourished thus her eggs just "wither away" or "dry up" before conception. A woman of average build and weight is best suited to conceive. Elderly Gikuyu healers reported that a woman with a protruding umbilicus (hernia), gikonyo, was highly admired by suitors since this portrayed her potential fertility. Traditionally the name ngonyo or wakonyo was a popular female nick-name denoting beauty and fertility.

To be "Kept", "bound" or "tied" was again mentioned and this was a common cause across various groups in Kenya such as the Kikuyu, Luo, Luyia and Kamba. Among the Luo and Luhya a grandmother plays a key role in fertility/infertility. It was explained by one participant that:

"Grandmothers used to take specially the undenwear or the soil which has your period and hide somewhere in the kitchen in the thatch by the fire hearth where smoke passes as a sign to prevent the girl from getting pregnant-- so in case she dies before she had told you, you might not get children"

Here to be "kept" served a dual and often a binary opposing purpose. For a young unmarried girl, to be 'kept' offered protection against unwanted pregnancy and the shame, dishonour and, disgrace that would accompany it as well as the possibility of losing out on a marriage partner. A girl who had a child out of wedlock could only marry a very old man. Although virginity was valued and it was expected that girls would avoid sex till marriage, this was only normative. In reality, it was acknowledged that young girls and boys 
experimented with sex. The most important thing was therefore to avoid pregnancy. To be "tied" or "kept" reinforced this. Thus, "keeping", in this context, was desired to preserve the honour of the girl and family by "protecting" her from pregnancy out of wedlock. However; it also meant that one had to cultivate a cordial relationship with the grandmother to ensure that she can "unbind" one at the opportune time. It is precisely for this reason that grandmothers were feared and respected at the same time to avoid invoking their wrath. However, these aside, there are also "outright enemies" who would "keep" a girl or woman simply to deny them conception and the joy of having a child and this is not. meant to be temporary. These are most feared as they cannot be identified and therefore cannot be appeased; unlike the known grandmother with whom one can make peace so long she is still alive.

This concept has parallels in other countries cited in literature. For example, Baasher et al (1983) discussed the traditional practices affecting the health of women and young children in the eastern Mediterranean region. He noted that delay of pregnancy due to infertility is a matter of deep concern. The first cause to be thought of and excluded is "mushahara" a supernatural harm caused to individuals in vulnerable states by other persons' violation of taboos. Prevention is through strict observation of taboos, and certain practices are deliberately carried out to maintain pregnancy and prevent abortion. Similarly, Inhorn (1994) reported that among the poor of Egypt, female infertility is often attributed to Kabsa (aiso know as mushahara), a form of 'boundary-crossing' by symbolically polluted individuals into the rooms of reproductively vulnerable women. When Kabsa occurs, it causes the 'binding' of women's reproductive bodies, threatening their future fertility, their husband's virile procreativity, and, ultimately, the social reproduction of Egyptian society at large. Because Kabsa is viewed as the leading cause of female infertility, an extremely socially stigmatizing condition in Egypt, it is greatly feared by women.

\section{Fertility-Infertility Dialectic and Women's Social Status}

Children are insurance for old age and assurance of personal and lineage immortality, and this propels the desire to procreate. In the traditional African setting, one important process of marriage is the payment of bride wealth by a man to the woman's family, to ensure the monopoly of her conjugal rights and ownership of the offspring of the marital union. A woman's social status, direction in life, economic 
achievement, well being and the very meaning of marital life hinges around her ability to give birth and rear children. This ability is therefore, seen as the true mark of womanhood and the pride of the man. A childless marital union is plagued with tension, social stigma, economic exploitation, and psychological pressures from the husband's relatives. Consequently, many childless women try all desperate attempts and follow every advice that may lead to their getting an offspring, or at least are seen to be pregnant.

In patriarchal societies, women will start off in a subordinate role as daughters; a position that is transferred to their husbands at marriage through the exchange of marriage payments. It is essentially a functional role with rights and obligations that are legitimized by the production of offspring. Demonstrable fertility is therefore of vital importance both for her, her husband and lineage. To establish a social base, if not a power base, a woman must bear children as a gateway to property access. Thus, even though property inheritance is through the male line, it is women as mothers who are custodians of property before it is passed on to their children. Whereas, it is argued in literature that fertility and the inability of women to control it does work against women's status and power, infertility on the other hand does erode it absolutely. Infertility denies women recognition regardless of their economic status. The impact is much more detrimental for a majority of women for whom childbearing would be the only source of recognition and self-esteem. Infertile women lamented the fact that being childless relegated them to the status of a "nobody" and were also regarded thus by their friends, husbands and the immediate family. It is only in one community, the Samburu, where infertile women get social and emotional support such as being given a child or children to take care of as her own.(UNFPA/Population Council, 2001).

In this section I, present some of the comments of infertile women in terms of what they thought of themselves as a result of their infertility and much more how they are treated and what these means to their lives.

In the discussion groups participants mentioned a derogatory term used to refer to infertile women: Kiswahili - Tasha; Kikuyu - Thata; Luo Lur; Kamba - Ngungu; Luhya - Mkumba. What is important is that all these terms are "hard",- reflecting masculinity. They are used to show the inverted of women identity who have failed to portray their femininity through childbearing. The infertile women in the discussion groups noted that these terms were often applied to them. The 
following are some of the excerpts from the participants' presentation of how these terms were used in various ways to insult and intimidate them; a constant reminder of their worthlessness:

"... Wherever you go it is insults. Even when you disagree
with somebody they know they will silence you by calling
you the name-tasha. When a child wrongs you, you cannot
report him because you have no child-you will be asked
"do you know anything about labour pains"? You will be told
to go and have your own. Discipline of children is the
exclusive privilege of those who have experienced
childbirth.

The link between production and reproduction is most evident when the fertility and infertility of a woman is considered. Immediately after marriage, it is expected that the woman will be well fed. However she has to reciprocate by bearing children as her contribution to the forces of production basically to maintain the balance of production and reproduction. A woman who does not fulfil her end of the bargain disrupts this tempo and she knows it. She also realizes what the looming consequences are likely to be. This is well articulated in the following excerpt:

If you get married in a place and they come to realize that you cannot have a baby, they chase you out of that boma (home) telling you that you are eating food for nothing... the benefit of marnying a woman is to get a child.

If you get married and fail to have a child your in-laws do not like you. I was two years without a child and my motherin-law used to say she does not want to live with a Mureithiherdsman

To be labelled a herdsman did summarily indicate that the young woman had failed to live to her role- that of childbearing.

The predicament of an infertile woman can also be reinforced by ideas generated from women who were fertile but were giving their views as to what it would mean to them if they had been infertile. One of the participants explained that she works hard because she has childrenshe would not work as hard if she had no children. This is evident in her comment: "Whom would I be working for?" This argument was 
repeated in a number of views from participants, emphasizing the notion of fulfilment and completeness that results from the mere fact of having a child. In contrast, they noted that a childless marriage is sheer torment, stressful for the woman and divorce is inevitable. One participant summarized the impact of infertility as follows: "Infertility leads to a lot of loneliness as one has no age group friends. -When mothers talk fondly about their children you have nothing to say- you can only be envious". In the communities studied women relate by age sets and in some, this was more marked by initiation rites that would move you to the next stage, often marriage and childbearing. Infertility disrupts this flow and a girl becomes a social misfit. Related to this is the comment that:

A child is a gift from God and you feel happy to get one. To be called the mother of so and so is beautiful. If children are bought, everyone could have one.

This reflects the deification of motherhood that is denied, with all the rewards that go with it that are all meaningful, a woman, the highest being one of recognition and security. The absence of a child literally blocks all these. In Layne's paper: Motherhood lost: Cultural dimensions of miscarriage and stillbirth in America (1990) in which grief experienced by the individual is legitimized and is similar to the experiences expressed by these women for whom motherhood is denied and with it the absence of a child and parenthood.

This signifies the idea that women acquire social status, recognition and indeed their personhood via childbearing. The situation of secondary infertility called kurugamira among the Kikuyu and kusingira among the Luhya denoting to stop, was contrasted with primary infertility. Infertile women wished at least to have had this status as it indicates that one has demonstrated fertility at some point in time. This also confers normality that one is like the others, an affirmation that is badly needed. This parallels the observation in the studies done in the US that the use of sonogram images and fetal monitors play an important role in pregnancy loss:

Sonogram photos and scraps from fetal monitors are frequently saved by bereaved parents and utilized as evidence to prove to others that a "baby" existed. (Layne 1992) 
Although infertility is overwhelmingly attributed to women, there are instances where it would be clearly caused by male. In a situation where it is established that it is the man who is infertile, the society is more accommodating. For example, among the Luo one informant explained that "the husband's parents give you the okay to choose a relative, for example his brother, classificatory brother or cousin, and this remains a secret". This, also happened among the Luhya and the Kikuyu where a woman was allowed to sleep with another man to get children for an infertile husband. Here social paternity was moreimportant than biological paternity. Besides, women had the opportunity to have children with other men, secretly. As one participant aptly explained:

\begin{abstract}
"Here, if it was a man who was infertile, the woman would go out of wedlock and get children. However, this was kept secret and even if the husband knew, one would not be asked. Some husbands chose who was to sleep with the wife so as to give the couple children. This was however a secret"
\end{abstract}

Thus while an infertile man is well cushioned and even protected from ridicule an infertile woman suffers displacement, has to bear the image of spoilt identity as is evident in the case of a woman referred to as Mureithi- a herdsman! The community's perception of infertility in various traditional beliefs cannot be overemphasized. The ability to procreate received praise and admiration. A woman who, for one reason or other, is unable to execute this role undergoes serious social stigma and this is deeply felt by the infertile woman. This is what (Whitehead and Gonzalez, 1995) have referred to as the hidden burden of infertility. It is internalized as a 'spoilt identity' a feeling of being inferior, degraded, deviant and shamefully different (Goffman, 1963). Emotional and even physical harassment by the male spouse or his relatives is common as is evident in the cases cited.

In patrilinial societies where the only avenue to accessing resources through children is the norm, an infertile woman is of no fixed abode. Again, this is demonstrated by the fertile woman who says, "a child makes you receive land- that is our inheritance". Children anchor women while a childless woman is rendered homeless: Throughout the discussions infertile women frequently referred to the fact that they had not 'settled' and this is often precipitated by being childless. 


\section{Conclusion}

Women world-wide appear to bear the major burden of reproductive setbacks of all kinds including blame for reproductive failure. This causes personal grief and frustration, marital stress, sometimes leading to divorce, social stigma and ostracism (Whiteford and Gonzalez, 1995; Inhorn, 1994; Inhorn and Buss, 1993). The views presented in this paper reflect this dilemma where blaming the victim is the norm. Infertility does compound and reinforce the already low social status of women. Thus women do not only face psychological problems but also the fact that the chances of being economically isolated are real. Hence, the women suffer double grief and jeopardy: the inability to bear children and the imminent loss of a livelihood that would otherwise be guaranteed by the bearing of children. For women who are infertile and poor it is total lose with no recourse. This calls for a more concerted effort to address issues of infertility through allocation of resources at the programme level. To date, infertility is covered under the broad spectrum of reproductive health but in practice little is done, as it is not seen as a major priority compared with fertility control. This is a situation where intervention should not only be based on magnitude in terms of numbers but a focus on individual suffering that is generated by infertility. Part of the intervention would be on service delivery at appropriate levels but also community education to demystify the problem in order to foster ideas on prevention and prompt health seeking.

\section{REFERENCES}

Davis-Floyd R.E., and Sargent C.F. (Eds.) 1997.

Childbirth and Authoritative Knowledge: Cross-Cultural Perspectives. University of California press, Berkeley,.

Ginsburg F.D., and Rapp R. (Eds.). 1995.

Conceiving the New World Order: The Global Politics of Reproduction. University of California press, Berkeley, .

Greenhalgh S. (Ed.) 1995.

Situating Fertility: Anthropology and Demographic Inquiry. Cambridge University

Press, Cambridge,.

Ericksen K. and T. Brunette. 1996.

Patterns and predictors of infertility among African women: a cross-national survey of twenty-seven nations. Soc. Sci. and Medicine, 42(2):209-20.

Family Care International. (Cairo, Sept. 1994) Action for the $21^{\text {st }}$ Century. 
Reproductive Health \& Rights for All. A summary Report on the. International Conference on Reproduction \& Develapment (ICPD)

Feldman-Savelsberg, P. 1994.

Plundered kitchens and empty wombs: Fear of infertility in the Cameroonian grassfields. Soc. Sci. \& Med. Vol.39: 4, 463-74,

Helman, C. G.1994.

Culture, Health and IIIness, Butterworth-Heinemann Ltd. London

Gerrits Trudie, 1997.

Social and Cultural aspects of Infertility in Mozambique. Patient Education and Counseling 31 (1997) 39-48.

Inhorn Marcia C. and Kimberly A. Buss. 1993.

Infertility, infection, and iatrogenesis in Egypt: The anthropological epidemiology of blocked tubes, Medical Anthropology, Vol.15, pp.217-44.

Inhorn, Marcia C. 1994.

Interpreting infertility: Medical anthropological perspectives. Soc. Sci. and Medicine, 39 (4):459-61.

Karen Ericksen and Tracy Brunette. 1996.

Patterns and predictors of infertility among African women: $A$ cross-national survey of twenty-seven nations. Soc. Sci. and Medicine, 42 (2):209-20.

Kenya National Reproductive Health Strategy for the Years 1997 to 2010: A Ministry of Health Policy document

(November 1996).

Kenya Demographic and Health Survey (DHS), 1993. (May 1994)

Kenya National Population Census, 1999

Kleinman, R.L and P. Senanayake. 1979.

Handbook on infertility. London, International Planned Parenthood Federation, $p$. 58.

Kleinman A. 1988

The Illness Narratives: Suffering, Healing and The Human Condition. Basic Books Inc. USA.

Layne, L. 1992.

"Of Fetuses and Angels: Fragmentation and integration in Narratives of Pregnancy Loss" in Knowledge and Society: The Anthropology of Science and Technology, Vol. 9 pp.29-58. JAI Press INC.

Layne', L. 1990.

Motherhood Lost: Cultural Dimensions of Miscarriage and Stillbith in America in Women \& Health Vol. 16(3/4). The Haworth Press, Inc.

L.eke, R.J, J.A. Oduma, S. Bassol-Mayagoita, et al. 1993.

Regional and geographical variations in infertility: effects of environmental, cultural and socio-economic factors. Environmental Health Perspectives. Vol. 101, Suppl. 2, pp. 73-80, Juiy.

Linda M. Whiteford and Lois Gonzalez. 1995.

Stigma: The hidden burden of infertility. Soc. Sci. and Medicine, 40 (1):27-36. 
MacCormack Carol (Ed.) 1994.

"Health, Fertility, and Birth in Moyamba District, Sierra Leone" In Ethnography of Fertility and Birth. $2^{\text {nd }}$ Edition, pp. 5-139. Prospect Heights, III, Waveland Press.

Mathews, T; J.K. Mati, J.N. Fomulu. 1981.

A study of infertility in Kenya; results of investigation of the infertile couple in Nairobi. East Afr. Medical Journal, 58(4):288- 97.

Mati J.K, G.E. Anderson, M.J. Carty, et al. 1973.

A second look into the problem of primary infertility in Kenya. East Afri. Medical Journal, 50(2):94-7.

Mati J. K. G. 1986.

Infertility in Africa; Magniture, major causes and approaches to management Obstet. Gynaecol E.C. Afr. 5: 65-69

Millar, M.I. 1987.

Genital chlamydia infection: A role for social scientists. Soc. Sci. and Medicine, 25(12):1289-99,.

Tarlatzis I. B.C.Tarlatzis, I.Diakogiannis, et al. 1993.

sychosocial impacts of infertility on Greek couples. Human Reproduction. 8(3):396-401. 